\title{
miR-141 is up-regulated in biopsies from Vietnamese patients with nasopharyngeal carcinoma
}

\section{Thuan Duc LAO(a) Truong Van NGUYEN(b) Dung Huu NGUYEN(c) Minh Trong NGUYEN(c) Chuong Hoang NGUYEN(d) Thuy Huyen Ai LE(a)}

(a) Ho Chi Minh City Open University, Faculty of Biotechnology, Department of Pharmaceutical Biotechnology, Ho Chi Minh City, Vietnam.

(b)Ho Chi Minh City International University, School of Biotechnology, Ho Chi Minh City, Vietnam.

(c) Cho Ray Hospital, Department of Otorhinolaryngology, Ho Chi Minh City, Vietnam.

(d) University of Science, Vietnam National University, Faculty of Biology and Biotechnology, Ho Chi Minh City, Vietnam.

Declaration of Interests: The authors certify that they have no commercial or associative interest that represents a conflict of interest in connection with the manuscript.

Corresponding Author:

Thuy Huyen Ai Le

E-mail: thuy.lha@ou.edu.vn

https://doi.org/10.1590/1807-3107bor-2018.vol32.0126

Submitted: December 16, 2017

Accepted for publication: October 16, 2018

Last revision: November 05, 2018

\begin{abstract}
Novel biomarkers for screening, diagnosis and monitoring the treatment of nasopharyngeal carcinoma (NPC), one of the most common cancers in Vietnam, are urgently required. Increasing evidence suggests that microRNA-141 (miR-141) is associated with NPC, owing to its ability to affect the expression of genes that modulate tumorigenesis. Unfortunately, research on miR-141 expression in Vietnamese patients is limited. Therefore, the objective of the current study was to evaluate miR-141 expression and assess whether miR-141 might be a potential biomarker for diagnosis of NPC in Vietnamese patients. Total RNA isolated from 40 NPC biopsy samples and 37 non-cancerous samples was analyzed by quantitative reverse-transcription PCR. The miR-141 expression levels were compared between NPC biopsy and non-cancerous samples. The frequency of miR-141 detection was $37.50 \%$ and $10.80 \%$ in the NPC and non-cancerous samples, respectively $(p=0.0143)$. The miR-141 expression was 5.27 times higher in tumor samples than non-cancerous samples. Additionally, the RR (Relative risk) and OR (Odds ratio) were $1.83(95 \% \mathrm{CI}=1.2576-2.6675, \mathrm{p}=0.0016)$ and $4.95(95 \% \mathrm{CI}=1.4625-16.7541, \mathrm{p}=0.01)$, respectively. In conclusion, miR-141 was up-regulated in the biopsy samples and thus may be a potential biomarker for NPC in the Vietnamese population.
\end{abstract}

Keywords: Biomarkers; Carcinoma; microRNAs; Biopsy; Nasopharynx

\section{Introduction}

Nasopharyngeal carcinoma (NPC), which has pronounced differences in distribution according to geography and ancestry, is the fifth most common cancer worldwide. NPC is present in 86,691 cases (age-standardized rate $(\mathrm{ASR})=1.2 / 100,000)$ and is responsible for $50,831(\mathrm{ASR}=0.7 / 100,000)$ deaths annually in Vietnam. ${ }^{1,2,3}$ Although patient diagnosis has improved in recent decades, most NPC patients are still diagnosed at an advanced stage of NPC, owing to ambiguous symptoms. ${ }^{4,5,6}$ Therefore, improved identification of potential biomarkers associated with NPC progression is essential for the development of new diagnosis strategies and promising therapies.

The use of NPC biopsy samples is considered the "gold standard" in NPC diagnosis. Beside NPC biopsy samples, other types of samples, such as peripheral blood, nasopharyngeal swabs, throat swabs, plasma and 
saliva have been used for detecting the etiological causes of NPC, including the presence of Epstein-Barr virus, methylation and microRNAs (miRNAs, denoted by the miR prefix) associated with NPC., ${ }^{7,9,10,11,12}$ In our recent study, we found that among EBNA-1, EBNA-2, LMP-1 and LMP-2, at least one EBV DNA was detectable in $57.9 \%$ of NPC samples, whereas a low frequency was found in non-cancerous swab samples..$^{13}$ Another recent study has also detected significantly elevated EBV DNA loads and miRNAs, such as mir-bart-5p, in nasopharyngeal swab samples from non-NPC patients. ${ }^{10,11,12}$

miRNAs are a small class of endogenous, functional non-RNAs that are highly conserved and are widely found in animals, plants, protists and viruses; miRNAs are involved in a wide range of biological processes, such as cell proliferation, differentiation, apoptosis and motility, through posttranscriptional repression of gene expression. ${ }^{6,14,15,16,17}$ miRNA-141 (miR-141) is a member of the oncogenic miRNAs clustered with miR-200c, located at 12p13.31 (nt: 6964097-6964191, [+]). The dysregulation of miR141 is associated with many human cancers, such as hepatocellular carcinoma, renal cell carcinoma, breast cancer, ovarian cancer and NPC. 6,18,19,20,21 However, only a few publications have described miR-141 profiles in NPC. Zhang et al. ${ }^{22}$ have reported that miR-141 is up-regulated in NPC specimens compared with normal nasopharyngeal epithelium, and that it affects the cell cycle, apoptosis, cell growth, migration and invasion in NPC cells. Additionally, targets of miR-141, including BRD3, $P T E N$ and $U B A P 1$, have been found to be involved in a gene-miR-141 network contributing to NPC development. ${ }^{22}$ Chen et al. ${ }^{23}$ have reported that miR141 is regulated by SPLUNC1 (short palate, lung and nasal epithelium clone 1) and is related to the differentiation stages of NPC cell lines; notably, miR141 expression is significantly elevated in NP-69 cells (a nasopharyngeal epithelial cell line) transfected with LMP1. ${ }^{23}$ Another report has indicated that the expression of miR-141 is markedly increased in NPC tissues and is involved in BRD7-medidated cell progression and tumor formation through suppression of the PTEN/AKT axis in nasopharyngeal carcinoma, thus providing several promising targets for NPC diagnosis. Furthermore, inhibitors specific to miR-141 may provide a promising strategy for NPC therapy. ${ }^{6}$

To our knowledge, no studies of the types of miR-141 expression in Vietnamese NPC patients have been reported to date. Therefore, the current study is the first case-control study on Vietnamese clinical NPC samples. The aim of the study was to access the differential expression of miR-141 in biopsy samples from NPC patients and non-cancerous nasopharyngeal swab samples to reveal potential biomarkers for NPC.

\section{Methodology}

\section{Ethics statement}

Institutional Ethics Board approval was obtained from the Medical Ethics Committee of the Cho Ray Hospital, Ho Chi Minh City, Vietnam (decision number 516/BVCR-HDDD). Collection of all samples used in this study was approved by the Cho Ray Hospital, and samples were obtained from all participants in the current study. The patients were required to provide signed informed consent forms.

\section{Sample collection}

A case-control study was carried out. A total of 40 NPC biopsy samples were archived from the Cho Ray Hospital, Vietnam. All samples were submitted to the histopathological department, and NPC was confirmed through immunohistochemistry. The mean age of the NPC patients was 49.6 (range from 15 to 72 ). The subjects included 31 males (accounting for 77.5\%) and nine females (accounting for $22.5 \%$ ). The NPC biopsy samples were confirmed as non-keratinizing squamous cell carcinoma and undifferentiated carcinoma via hematoxylin and eosin staining in histological examination (Figure 1). For non-cancerous controls, 37 nasopharyngeal swab samples that were negative for nasopharyngeal carcinoma were collected from non-NPC patients. All samples were placed in 1.5-ml tubes containing PBS buffer and stored at $-20^{\circ} \mathrm{C}$ for further experiments.

RNA, including miRNAs, was isolated from NPC biopsy samples and non-cancerous samples 

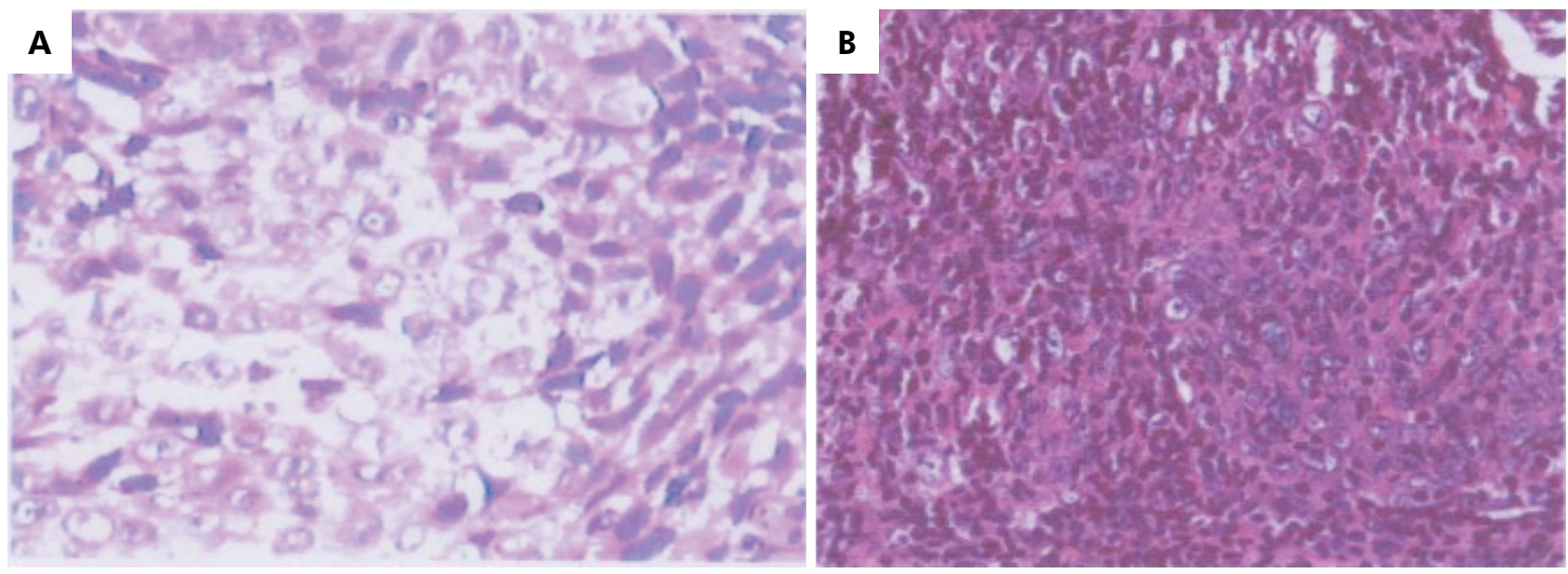

Figure 1. Histological examination of (A) undifferentiated carcinoma (WHO type 3) and (B) non-keratinizing squamous cell carcinoma (WHO type 2).

with a mirVana ${ }^{\mathrm{TM}}$ miRNA Isolation Kit (Ambion, Life Technology) according to the manufacturer's instructions. cDNA was reverse transcribed from approximately $5 \mathrm{ng}$ of Total RNA with a SensiFAST ${ }^{\mathrm{TM}}$ cDNA synthesis Kit (Bioline). The detection of miR-141 was performed with a quantitative real-time PCR (qRT PCR) assay with Taqman ${ }^{\mathrm{TM}}$ Advanced Advance miRNA kits (Thermo Fisher Scientific). Five microliters of the RT reaction product was used in each reaction for detection of miR-141. The UniSp6 rRNA was used as the internal control to normalize $\mathrm{Ct}$ values, because of the non-differential expression in tumor and adjacent healthy samples.

\section{Statistical analysis}

Data were analyzed in Medcalc ${ }^{\circledR}$ Version 12.7.0.0. All $p$ values were two-sided, and values less than 0.05 were considered significant. All values are reported as mean $\pm \mathrm{SD}$. The relative expression of miR-141, as determined with q-PCR, was analyzed with the $2^{-\Delta \Delta C t}$ method. Values greater and less than one were used to indicate up-regulation and down-regulation, respectively. Chi square tests were used to determine the association between miR-141 expression and NPC status. Moreover, the association between the expression of miR-141 and NPC risk was estimated by computing the OR, $\mathrm{RR}$ and 95\% confidence intervals (CI).

\section{Results}

First, UniSp6 rRNA was detected in both case and control samples through real-time PCR. Both the NPC biopsies and non-cancerous controls were positive for UniSp6 RNA (Figure 2). The mean Ct values for UniSp6 rRNA in the case and control groups were $29.60 \pm 0.56$ and $30.24 \pm 0.46$, respectively. Therefore, UniSp6 was suitable as a reference gene (internal control) to normalize miR-141 expression between the case and control groups.

In the current case-control study, miR-141 expression in the NPC biopsy samples was positive in $37.50 \%$ ( 15 of 40 cases) and negative in $62.50 \%$ ( 25 of 40 cases). In the non-cancerous samples, miR-141 expression was positive in $10.80 \%$ (4 of 37 cases) and negative in $89.20 \%$ (33 of 37 cases). The expression of miR-141 in NPC biopsy samples was significantly higher than that in non-cancerous samples $(p=0.0143)$. The mean Ct values of miR-141 in the case and control groups were $26.27 \pm 0.95$ and $29.50 \pm 1.56$, respectively. Relative quantification of miR-141 expression between the case and control groups was determined with the $2^{-\Delta \Delta \mathrm{Ct}}$ method. The miR-141 level was 5.28 times higher in tumor samples than in non-cancerous samples $\left(2^{-\Delta \Delta \mathrm{Ct}}=5.28(3.16-8.82)\right)$ (Figure 3). Additionally, the OR and RR values computed between the expression of miR-141 and NPC were $4.95(95 \% \mathrm{CI}=1.4625-16.7541, \mathrm{p}=0.01)$ and $1.83(95 \% \mathrm{CI}=1.2576-2.6675, \mathrm{p}=0.0016)$, respectively. 
A

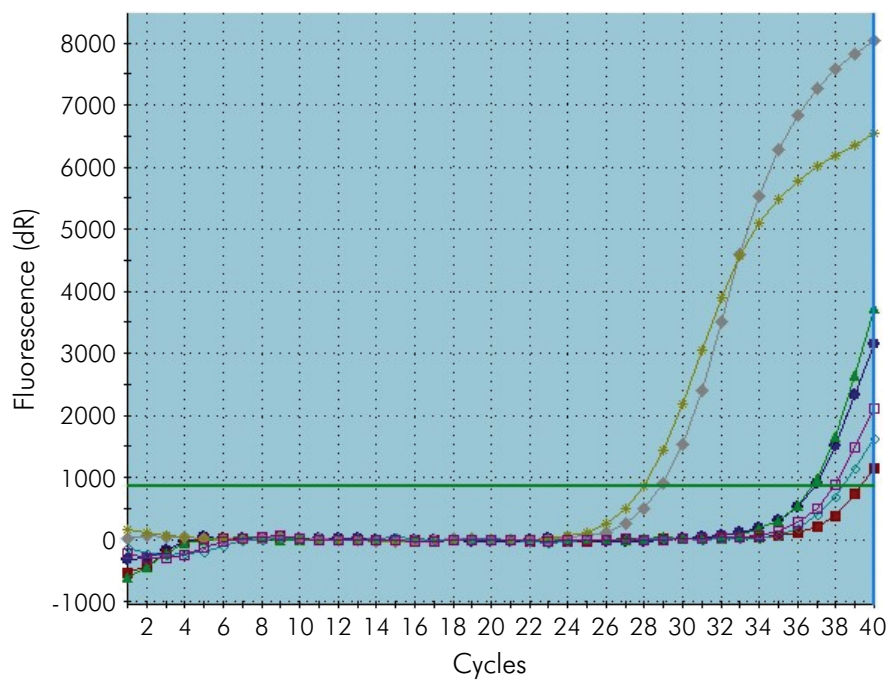

B

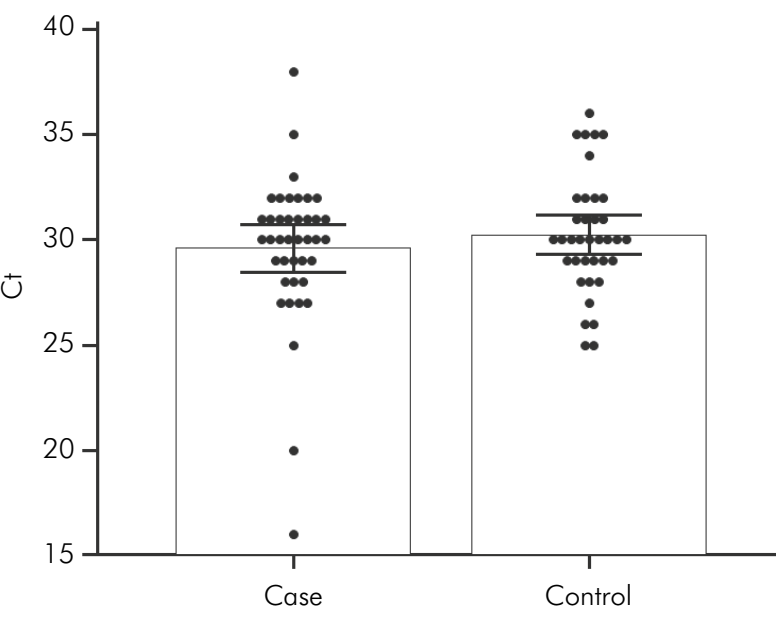

Figure 2. (A) UniSp6 rRNA expression detected in a representative healthy control. (B) The mean Ct values (mean \pm SE) of UniSp6 rRNA in the case and control groups. Each black dot indicates the Ct value of each sample.

A

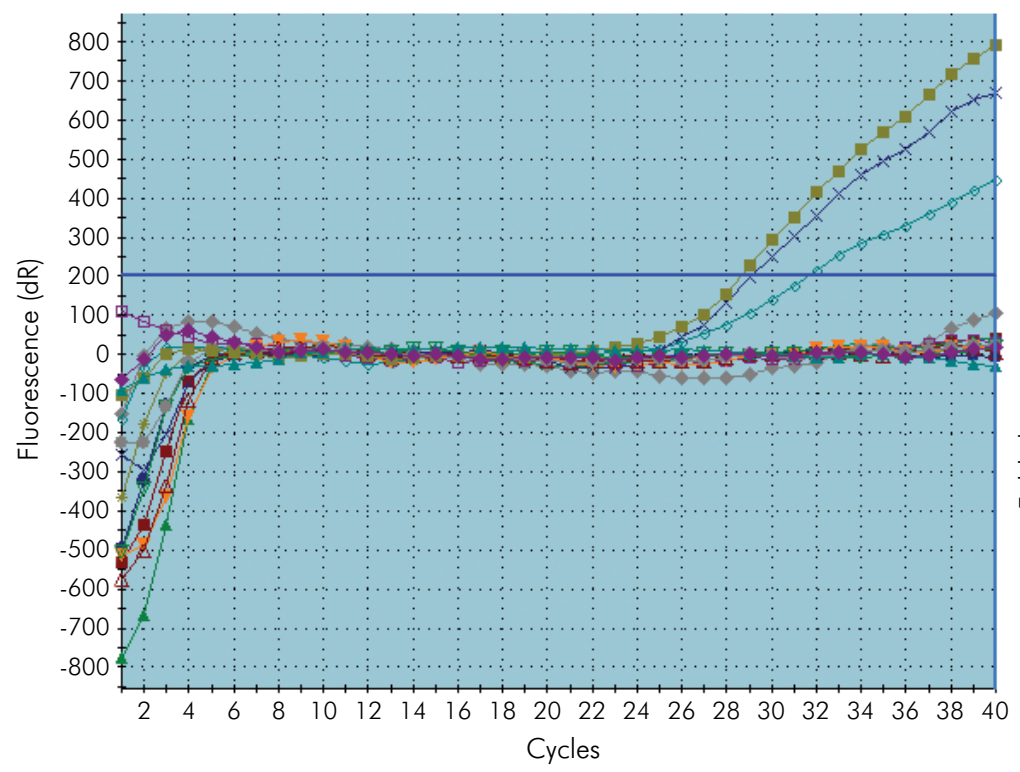

B

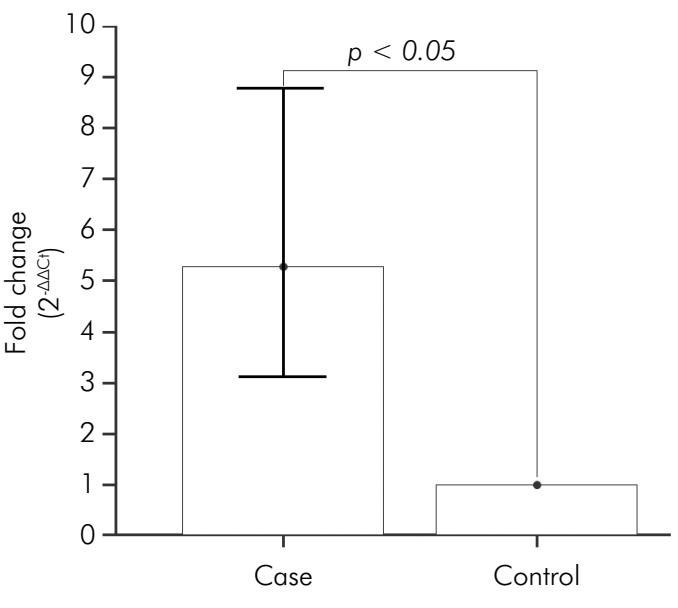

Figure 3. (A) miR-141 expression detected/undetected in representative NPC biopsy samples. (B) qRT-PCR showing up-regulation of miR-141 in NPC biopsy samples compared with non-cancerous samples.

\section{Discussion}

The amplification of nucleic acids from nasopharyngeal swabs and throat swabs has been found to be suitable for all types of testing, owing to stability at room temperature and in storage at $4^{\circ} \mathrm{C} .{ }^{10}$ Additionally, according to the $\mathrm{WHO}$, many types of swab specimens, including nasopharyngeal swabs, are standard protocols used to obtain samples for 
clinical and quarantine station diagnosis. The use of nasopharyngeal swabs has many advantages in studying the expression of potential miRNA biomarkers in throat swabs of healthy control and NPC patients. ${ }^{11}$ In the current study, we investigated patients who underwent nasopharyngeal biopsy with imaging and biopsy results, confirmed the samples for NPC and performed an initial evaluation of miR141 expression in Vietnamese NPC patients. Many studies have demonstrated that dysregulation of miRNAs is associated with the pathogenesis of various human tumors. Recently, a distinct miRNA signature has been proposed as a potential biomarker for the diagnosis of various types of human cancer. ${ }^{6}$ Previous studies have demonstrated that the use of miRNAs, a class of non-protein encoding RNA molecules that function in epigenetic regulation, are a novel class of therapeutic targets for the treatment of cancer. ${ }^{24,25}$ One of them, miR-141, has been well documented to have both oncogenic and tumor suppressive roles in human malignancies. The oncogenic role has been observed in ovarian cancer and NPC. ${ }^{6,22,23,26,28}$ In the current study, we detected positive miR-141 expression in $37.50 \%$ of NPC biopsy samples (sensitivity $=37,50 \%$ ), thus suggesting that $37.50 \%$ of NPC samples can be expected to be positive for miR-141 expression. Moreover, the miR-141 expression in the NPC samples was higher than that in non-cancerous samples $(\mathrm{p}=$ 0.0143 ), thus indicating that the expression of miR141 is strongly associated with NPC. Additionally, the miRNA expression was confirmed to be 5.27 times higher in tumor samples than non-cancerous samples $\left(2^{-\Delta \Delta C t}=5.27\right)$. Up-regulated expression of miR141 was observed in NPC samples, as has also been observed in other populations and NPC cell lines. Up-regulated expression of miR-141 has been reported by Zhang et al., ${ }^{22}$ Chen et al. ${ }^{23}$ and Liu et al. ${ }^{6}$ in NPC tissue samples collected from The Second Xiangya Hospital of Central South University (Changsha, China) and The Second Xiangya Hospital and Hunan Province Tumor Hospital, and in NPC cell lines, as compared with non-cancerous samples. However, in Vietnam, no studies on miR-141 in NPC has been reported. This is a follow-up study based on previous findings regarding the use of biopsies to assess miRNA properties. The main difference between this study and previous publications is that we focused on the Vietnamese population, the country with the high mortality from NPC worldwide. The up-regulation of miR-141 was strongly correlated with NPC risk, and the results were significant on the basis of the RR and OR ( $p$ < 0.05). miR-141 highly associated with higher odds, counting for 4.95 times, of NPC cancer $(\mathrm{OR}=4.95,95 \% \mathrm{CI}=1.4625-16.7541, \mathrm{p}=0.01)$. Moreover, the expression of miR-141 was 1.83 times higher than no miR-141 expression in NPC $(\mathrm{RR}=1.83,95 \% \mathrm{CI}=$ 1.2576-2.6675, $\mathrm{p}=0.0016$ ). Therefore, even though limited samples were analyzed in the current study, we investigated the potential differential expression of miR-141 in NPC biopsy samples and healthy nasopharyngeal swabs. Finally, we suggest that, in agreement with findings from previous studies, miR141 is up-regulated and has an oncogenic role in NPC development, including in the Vietnamese population; thus, it is a potential marker for NPC in Vietnamese NPC patients. In further studies, miR-141 obtained from non-invasive specimens, such as throat swabs or nasopharyngeal swabs, will be investigated as a biomarker for the screening and early diagnosis of NPC in the Vietnamese population.

\section{Conclusion}

In the present study, the potential differential expression of miR-141 in NPC biopsy samples and nasopharyngeal swabs from healthy individuals was investigated. The miRNA was detected in $37.50 \%$ of cancerous and $10.80 \%$ of non-cancerous samples $(\mathrm{p}=$ 0.0143 ). The present study demonstrated that miR-141 is up-regulated 5.25 fold in NPC samples compared with non-cancerous samples in the Vietnamese population. Therefore, miR-141 may be a potential diagnostic biomarker for NPC in Vietnamese patients. To develop a non-invasive method for clinical applications in screening and early diagnosis of NPC, further investigations should be performed in larger groups of samples as well as non-invasive samples.

\section{Acknowledgments}

We wish to express our thanks for the research project sponsored by the Ministry of Education and Training, Vietnam; Ho Chi Minh City Department 
of Science and Technology, Vietnam; Ho Chi Minh City Open University, HCMC, Vietnam. We also thank all the recruited participants in this work and all the otorhinolaryngology staff members of in Cho Ray Hospital, Ho Chi Minh City, for collecting the samples used in these studies.

\section{References}

1. Pathmanathan R, Prasad U, Sadler R, Flynn K, Raab-Traub $N$. Clonal proliferations of cells infected with Epstein-Barr virus in preinvasive lesions related to nasopharyngeal carcinoma. N Engl J Med. 1995 Sep;333(11):693-8. https://doi.org/10.1056/NEJM199509143331103

2. Costa VG, Marques-Silva AC, Moreli ML. The Epstein-Barr virus latent membrane protein-1 (LMP1) 30-bp deletion and Xhol-polymorphism in nasopharyngeal carcinoma: a metaanalysis of observational studies. Syst Rev. 2015 Apr;4(1):46. https://doi.org/10.1186/s13643-015-0037-z

3. Dai W, Zheng H, Cheung AK, Lung ML. Genetic and epigenetic landscape of nasopharyngeal carcinoma. Linchuang Zhongliuxue Zazhi. 2016 Apr;5(2):16. https://doi.org/10.21037/cco.2016.03.06

4. Epstein JB, Jones CK. Presenting signs and symptoms of nasopharyngeal carcinoma. Oral Surg Oral Med Oral Pathol. 1993 Jan;75(1):32-6. https://doi.org/10.1016/0030-4220(93)90402-P

5. Hao SP, Tsang NM, Chang KP, Ueng SH. Molecular diagnosis of nasopharyngeal carcinoma: detecting LMP-1 and EBNA by nasopharyngeal swab. Otolaryngol Head Neck Surg. 2004 Nov;131(5):651-4. https://doi.org/10.1016/i.otohns.2004.04.013

6. Liu Y, Zhao R, Wang H, Luo Y, Wang X, Niu W et al. miR-141 is involved in BRD7-mediated cell proliferation and tumor formation through suppression of the PTEN/AKT pathway in nasopharyngeal carcinoma. Cell Death Dis. 2016 Mar;7(3):e2156. https://doi.org/10.1038/cddis.2016.64

7. Breda E, Catarino RJ, Azevedo I, Lobão M, Monteiro E, Medeiros R. Epstein-Barr virus detection in nasopharyngeal carcinoma: implications in a low-risk area. Rev Bras Otorrinolaringol (Engl Ed). 2010 May-Jun;76(3):310-5. https://doi.org/10.1590/S1808-86942010000300007

8. Liu X, Luo HN, Tian WD, Lu J, Li G, Wang L et al. Diagnostic and prognostic value of plasma microRNA deregulation in nasopharyngeal carcinoma. Cancer Biol Ther. 2013 Dec;14(12):1133-42. https://doi.org/10.4161/cbt.26170

9. Zheng XH, Lu LX, Li XZ, Jia WH. Quantification of Epstein-Barr virus DNA load in nasopharyngeal brushing samples in the diagnosis of nasopharyngeal carcinoma in southern China. Cancer Sci. 2015 Sep;106(9):1196-201. https://doi.org/10.1111/cas.12718

10. Bursle E, Robson J. Non-culture methods for detecting infection. Aust Prescr. 2016 Oct;39(5):171-5. https://doi.org/10.18773/austprescr.2016.059
11. Peng F, He J, Loo JF, Yao J, Shi L, Liv C et al. Identification of microRNAs in Throat Swab as the Biomarkers for Diagnosis of Influenza. Int J Med Sci. 2016 Jan;13(1):77-84. https://doi.org/10.7150/ijms.13301

12. Zheng XH, Lu LX, Cui C, Chen MY, Li XZ, Jia WH. EpsteinBarr virus mir-bartl $-5 p$ detection via nasopharyngeal brush sampling is effective for diagnosing nasopharyngeal carcinoma. Oncotarget. 2016 Jan;7(4):4972-80. https://doi.org/10.18632/oncotarget.6649

13. Lao TD, Nguyen DH, Nguyen TM, Le TA. Molecular Screening for Epstein-Barr virus (EBV): detection of Genomic EBNA-1, EBNA-2, LMP-1, LMP-2 among Vietnamese patients with nasopharyngeal brush samples. Asian Pac J Cancer Prev. 2017 Jun;18(6):1675-9. https://doi.org/10.22034/APJCP.2017.18.6.1675

14. Lee RC, Feinbaum RL, Ambros V. The C. elegans heterochronic gene lin-4 encodes small RNAs with antisense complementarity to lin-14. Cell. 1993 Dec;75(5):843-54. https://doi.org/10.1016/0092-8674(93)90529-Y

15. Croce CM, Calin GA. miRNAs, cancer, and stem cell division. Cell. 2005 Jul;122(1):6-7. https://doi.org/10.1016/i.cell.2005.06.036

16. Filipowicz W, Bhattacharyya SN, Sonenberg N. Mechanisms of post-transcriptional regulation by microRNAs: are the answers in sight? Nat Rev Genet. 2008 Feb;9(2):102-14. https://doi.org/10.1038/nrg2290

17. Ha M, Kim VN. Regulation of microRNA biogenesis. Nat Rev Mol Cell Biol. 2014 Aug;15(8):509-24. https://doi.org/10.1038/nrm3838

18. Chen X, Wang X, Ruan A, Han W, Zhao Y, Lu X et al. miR-141 is a key regulator of renal cell carcinoma proliferation and metastasis by controlling EphA2 expression. Clin Cancer Res. 2014 May;20(10):2617-30. https://doi.org/10.1158/1078-0432.CCR-13-3224

19. Lin L, Liang $H$, Wang $Y$, Yin $X, H u Y$, Huang J et al. microRNA-141 inhibits cell proliferation and invasion and promotes apoptosis by targeting hepatocyte nuclear factor-3 $\beta$ in hepatocellular carcinoma cells. BMC Cancer. 2014 Nov;14(1):879. https://doi.org/10.1186/1471-2407-14-879

20. Wu SM, Ai HW, Zhang DY, Han XQ, Pan Q, Luo FL et al. MiR-141 targets ZEB2 to suppress HCC progression. Tumour Biol. 2014 Oct;35(10):9993-7. https://doi.org/10.1007/s13277-014-2299-9 
21. Choi SK, Kim HS, Jin T, Hwang EH, Jung M, Moon WK. Overexpression of the miR-141/200c cluster promotes the migratory and invasive ability of triple-negative breast cancer cells through the activation of the FAK and PI3K/AKT signaling pathways by secreting VEGF-A. BMC Cancer. 2016 Aug;16(1):570. https://doi.org/10.1186/s12885-016-2620-7

22. Zhang L, Deng T, Li X, Liu H, Zhou H, Ma J et al. microRNA-141 is involved in a nasopharyngeal carcinomarelated genes network. Carcinogenesis. 2010 Apr;31(4):55966. https://doi.org/10.1093/carcin/bgp335

23. Chen P, Guo X, Zhou H, Zhang W, Zeng Z, Liao $Q$, et al. SPLUNC1 regulates cell progression and apoptosis through the miR-141-PTEN/p27 pathway, but is hindered by LMP1. PLoS ONE. 2013;8(3):e56929.

https://doi.org/10.1371/journal.pone.0056929

24. Kala R, Peek GW, Hardy TM, Tollefsbol TO. MicroRNAs: an emerging science in cancer epigenetics. J Clin Bioinforma. 2013 Mar;3(1):6. https://doi.org/10.1186/2043-9113-3-6

25. Shah MY, Ferrajoli A, Sood AK, Lopez-Berestein G, Calin GA. microRNA Therapeutics in Cancer - An Emerging Concept. EBioMedicine. 2016 Oct;12:34-42. https://doi.org/10.1016/j.ebiom.2016.09.017

26. Jaarsveld MT, Helleman J, Boersma AW, Kuijk PF, licken WF, Despierre $E$ et al. miR-141 regulates KEAP1 and modulates cisplatin sensitivity in ovarian cancer cells. Oncogene. 2013 Sep;32(36):4284-93. https://doi.org/10.1038/onc.2012.433 